\title{
Author Index, Volume 91
}

\author{
Abbott CM 136 \\ Alström-Rapaport C 293 \\ Antolin MF 373 \\ Araki M 510 \\ Arctander P 361 \\ Árnason E 557 \\ Asenjo A 202 \\ Auckland LD 584 \\ Ayre DJ 475
}

Backeljau T 239, 487

Bandou E 181

Bar-Hen A 528

Barragán MJL 232

Barth S 36

Barton NH 136

Barwegen MW 565

Beardmore JA 545

Beaumont AR 354

Bellido A 224

Berdnikov VA 577

Bickmore W 93

Birley AJ 217

Björklund M 481

Blanc JM 255

Bogdanova VS 577

Bouharmont P 528

Boulding EG 60

Bourguet D 78

Bradley DG 43

Brakefield PM 17

Brand J 389

Breiman A 2

Brennan AC 502

Breuker CJ 17

Brugmans B 494

Bullejos M 232

Busimi AK 36

Byrne M 389

Cameron ND 494

Capy P 440

Carareto CMA 570

Caron H 181

Carracedo MC 202

Casares P 202

Charlesworth D 262

Charlwood JD 407

Chiu C-H 3

Cilas C 528

Conte R 401

Cooper DW 153

Coosemans M 487

Dane F 314

David JR 440

de la Guardia RD 232

de Meaux J 345

Denholm I 98

Devine GJ 98
Devlin B 537

Donnelly MJ 407

Dorn S 430

do Rosário VE 407

Duce I 98

Dulout FN 248

Eldridge MDB 153

England PR 475

Ennos RA 193

Fernández Iriarte PJ 51

Fernández R 232

Ferreira C 407

Flintham JE 94

Förg-Brey B 136

Foster SP 98

Freeman AR 43

Frias Martins AM 239

Friedrich Utz H 36

Fromm M 337

$\mathrm{Fu} \mathrm{Y} 314$

Fuller DQ 43

Furlong RF 533

Gaillard C 43

Gair J 78

Gautschi B 9

Gibert P 440

Gil V 407

Giovambattista G 248

Gordon IL 85

Gorel FL 577

Gotoh T 208

Gottlieb LD 91

Gouin N 70

Grandjean F 70

$\mathrm{Gu} \mathrm{H} 430$

Guillemaud T 143

Guiller A 224

Gustafsson S 284

Haerty W 440

Hammer S 125

Hämmerli A 448

Hansen LB 276

Harper JA 396

Harris SA 95, 502

Hasson ER 51

Hattemer $\mathrm{HH} 337$

Heath M 322

Heimpel GE 373

Hiscock SJ 502

Hofman S 136

Hollocher H 28

Holsinger KE 1

Hong X-Y 208

Huang H 314

Isaacs H 539
Jiggins FM 382

Jordaens K 239

Jørgensen RB 276

Karamoto N 331

Kaulfus D 125

Kauserud H 163

Kearsey MJ 456

Keeling MJ 382

Kellman M 307

Kosterin OE 577

Kovarik A 268

Kremer A 181

Kumar P 43

Lagercrantz U 293

Lanaud C 322

Lang P 314

Lascoux M 293, 465

Latouche-Hallé C 181

Leach KA 584

Leitch AR 268

Lenstra JA 565

Lim KY 268

Lirón JP 248

Liu CM 4

Loftus RT 43

Lönn M 284

Macdonald B 389

Maclean A 136

Madec L 224

Madsen T 112

Maki M 300

Malta-Vacas J 407

Marchal JA 232

Martínez S 232

Maruyama K 510

Matsumura S 300

Mattice M 78

Matyasek R 268

McConnell KC 193

McCoy KD 422

McIntyre JD 255

McMahon WM 540

Melchinger AE 36

Merilä J 117

Michalakis Y 422

Mieuzet L 143

Miller NJ 217

Mirol PM 248

Mitchell-Olds T 345

Miyamoto N 331

Moreteau B 440

Morgan WG 396

Motamayor JC 322

Müller JP 9

Muwanika VB 361 
Nijman IJ 565

Noda H 208

Nodari RO 401

Norry FM 51

Núñez-Farfán J 415

Nürnberger B 136

Nyakaana S 361

Ode PJ 373

O'Hara RB 117, 373

Ohba K 510

Oliver B 535

Olsson M 112

Overall ADJ 217

Pain S 70

Pakkasmaa S 117

Palmé AE 465

Peleman J 494

Perry MD 544

Petrarca V 407

Pinto J 407

Pither R 307

Pooni HS 456

Poschlod P 519

Praetzel G 136

Promislow DEL 546

Ramboer A 181

Read JM 382

Reisch C 519

Reusch TBH 448

Reynolds J 70

Reynolds MM 584

Risterucci AM 322

Roden C 565

Roderick HW 396

Roeder K 537

Roelants P 487
Romero Mendoza L 565

Rönnberg-Wästljung A 293

Rousset F 107

Ruiz MT 570

Ruiz-Montoya L 415

Sainz A 28

Sánchez A 232

Schaschl H 125

Schmid B 9

Schumacher T 163

Scotting PJ 5

Sedrez dos Reis M 401

Semerikov V 293, 465

Semple CAM 542

Shimatani K 173

Shore JS 307

Shuker DM 6

Shykoff JA 9

Siegismund HR 276, 361

Sigurgíslason H 557

Simon J-C 143

Simon RC 255

Skibinski DOF 354

Smith VS 96

Snoke MS 546

Sousa CA 407

Souty-Grosset C 70

Strand MR 373

Suchentrunk F 125

Susilawati T 565

Syed NH 456

Szymura JM 136

Takahashi M 173, 331

Tani N 510

Tao WJ 60

Tatchell GM 217

Thomas HM 396

Tirard C 422
Tomaru N 510

Trung HD 487

Tsarouhas V 293

Tsumura Y 510

Turner G 354

Uchida K 510

Uller T 112

Van Bortel W 487

van Eijk MJT 494

Van Riel P 239

Vargas J 415

Vencovsky R 401

Verhagen R 239

Verkaar ELC 565

Vervaecke H 565

Vieira CP 262

Vieira J 262

Volpi E 437

Wain HM 353

Wang Q 430

Wasserman L 537

Whelan RJ 475

Whitlock MC 78

Wilder JA 28

Williams CG 584

Williamson MS 98

Wingender R 519

Wolf JB 438

Wolf M 28

Wood AR 354

Yamashiro T 300

Yang Z 533

Yoshimaru H 510

Young S 98

Zenger KR 153 